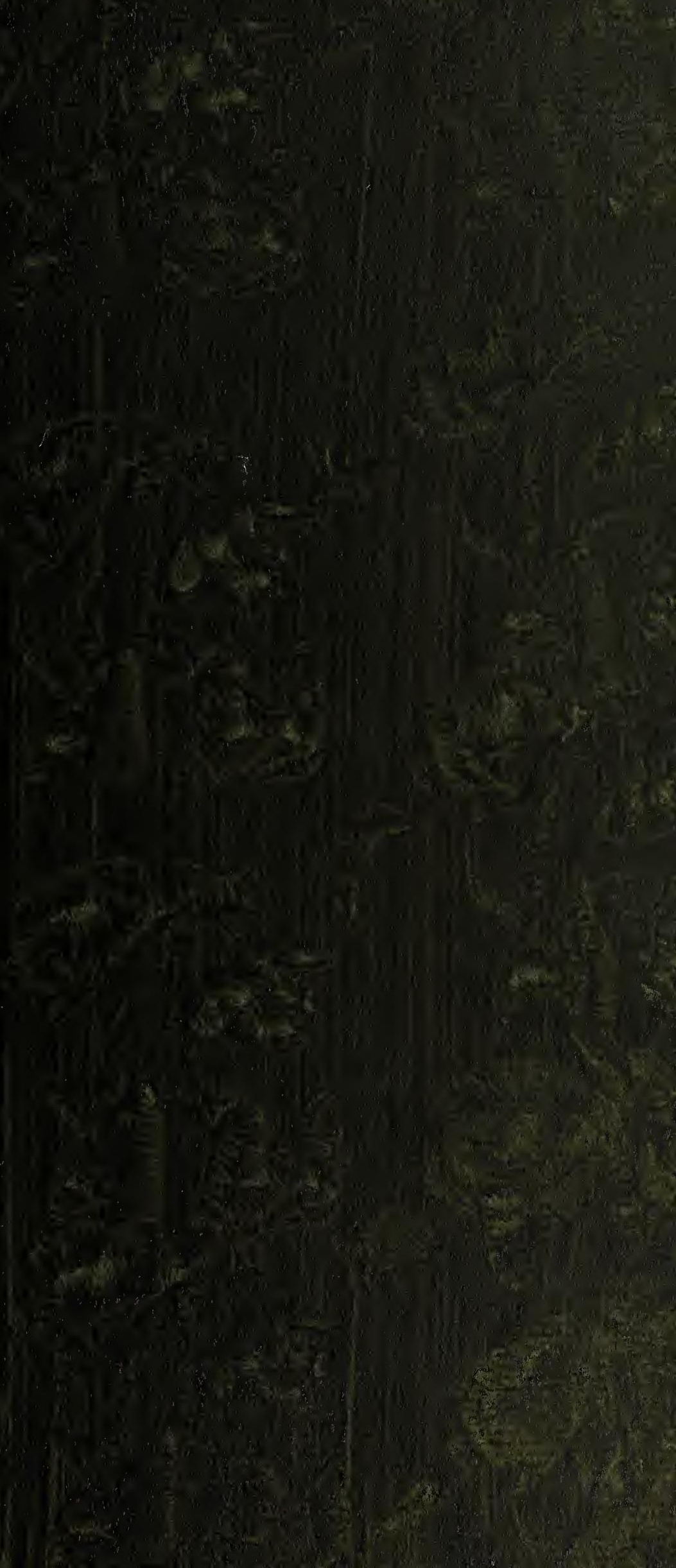


C.S.RAFINESQUE

Zoological rbotanical papers -

Mro"the Fleasures and duhis of wealct - 




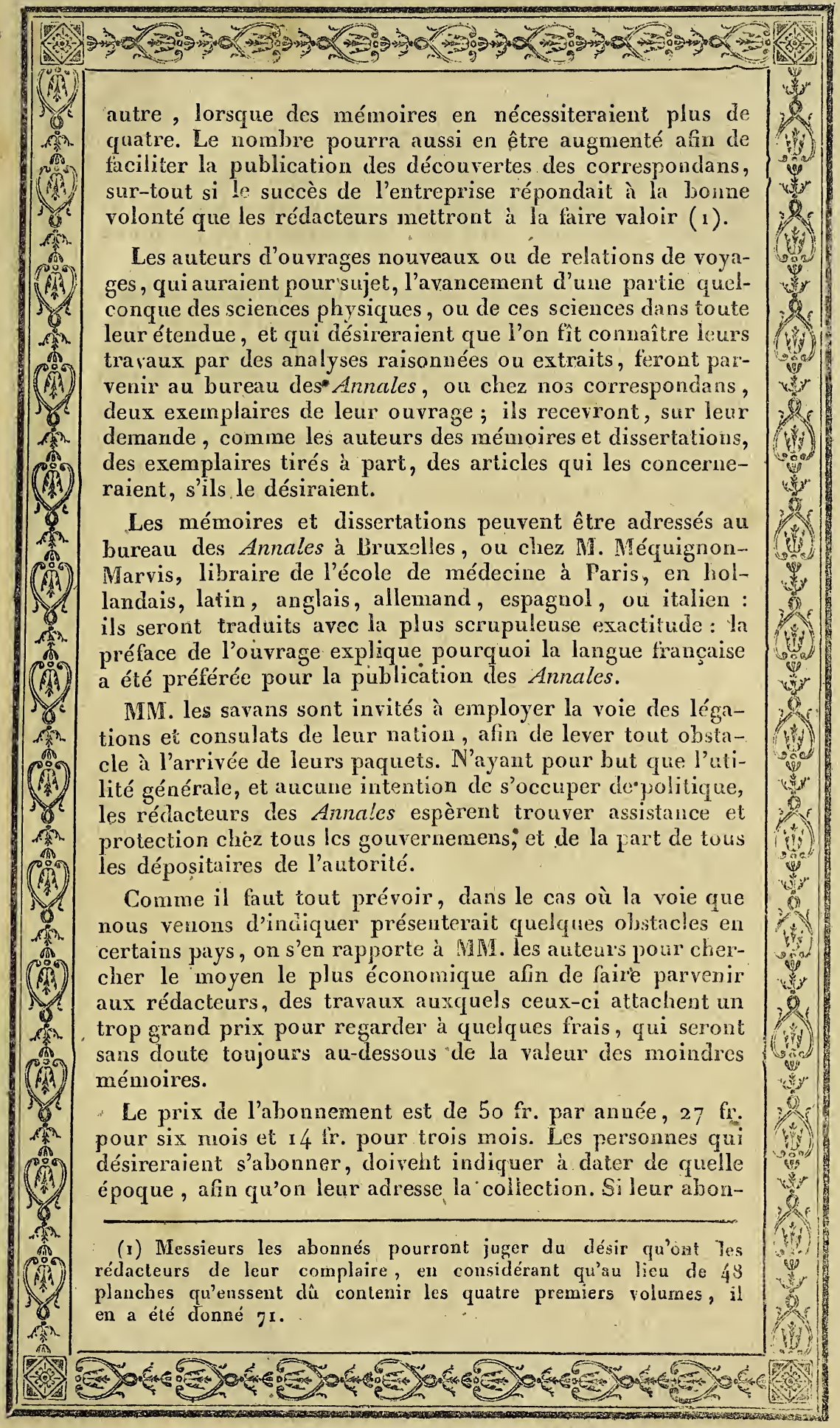




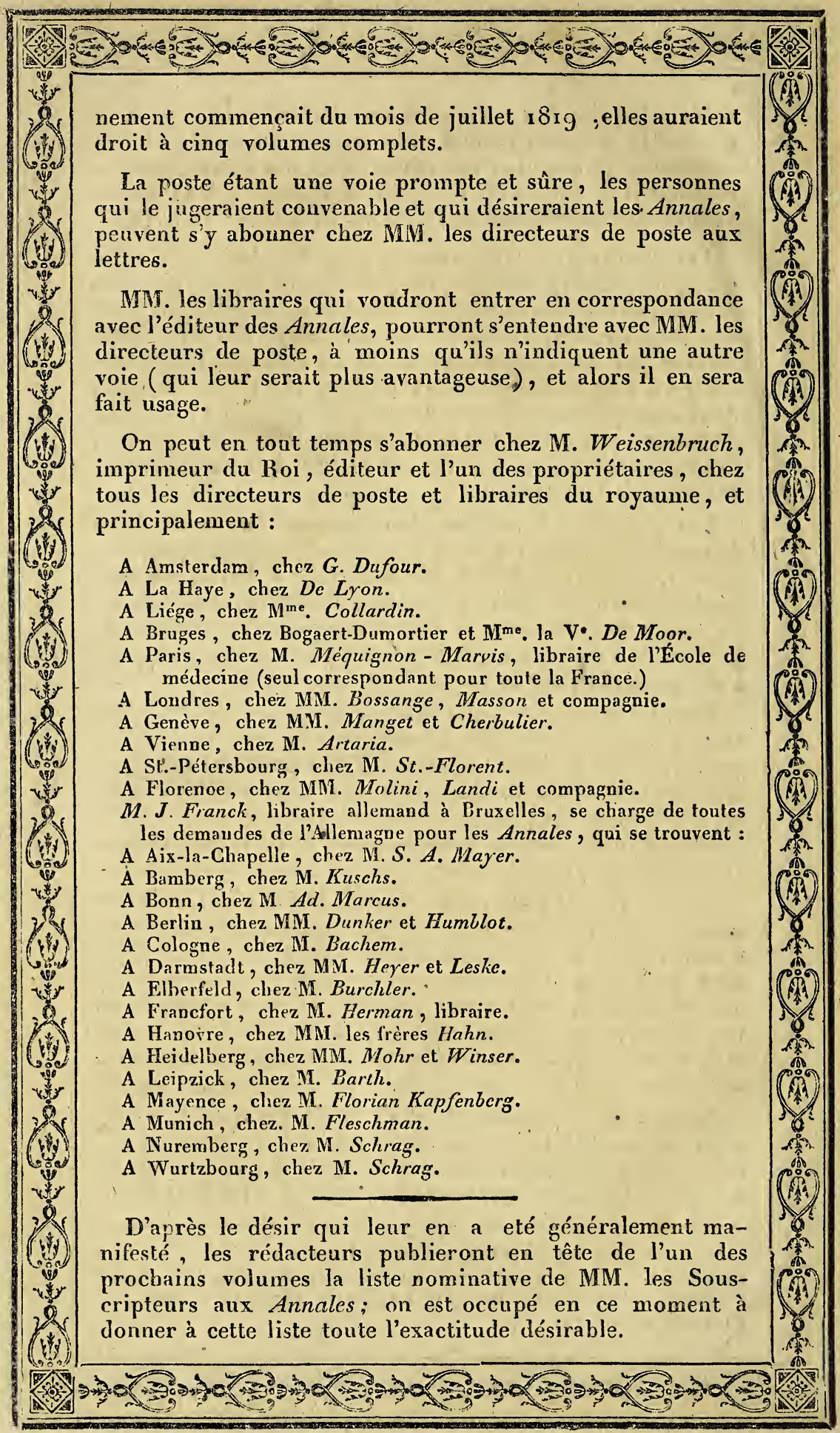




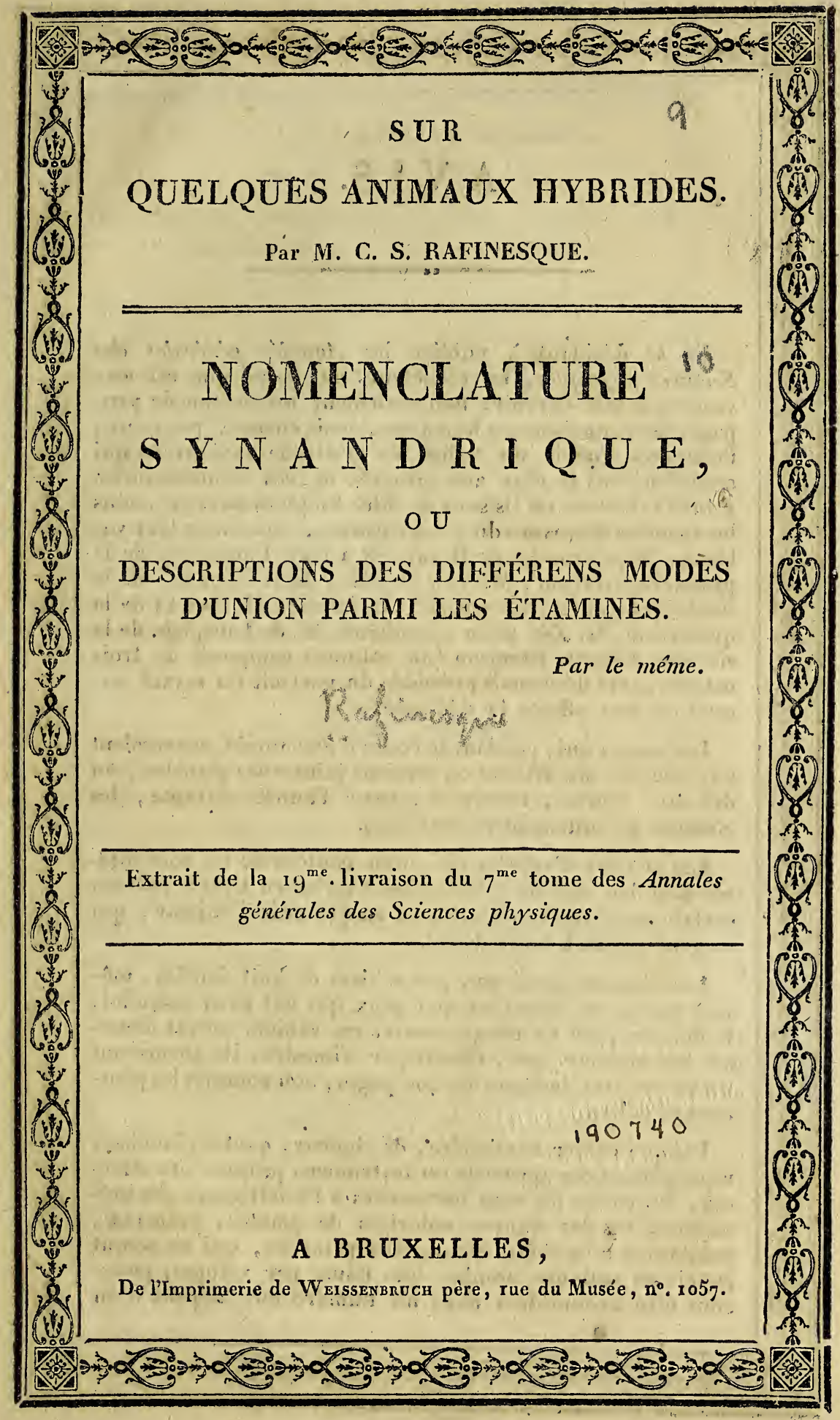




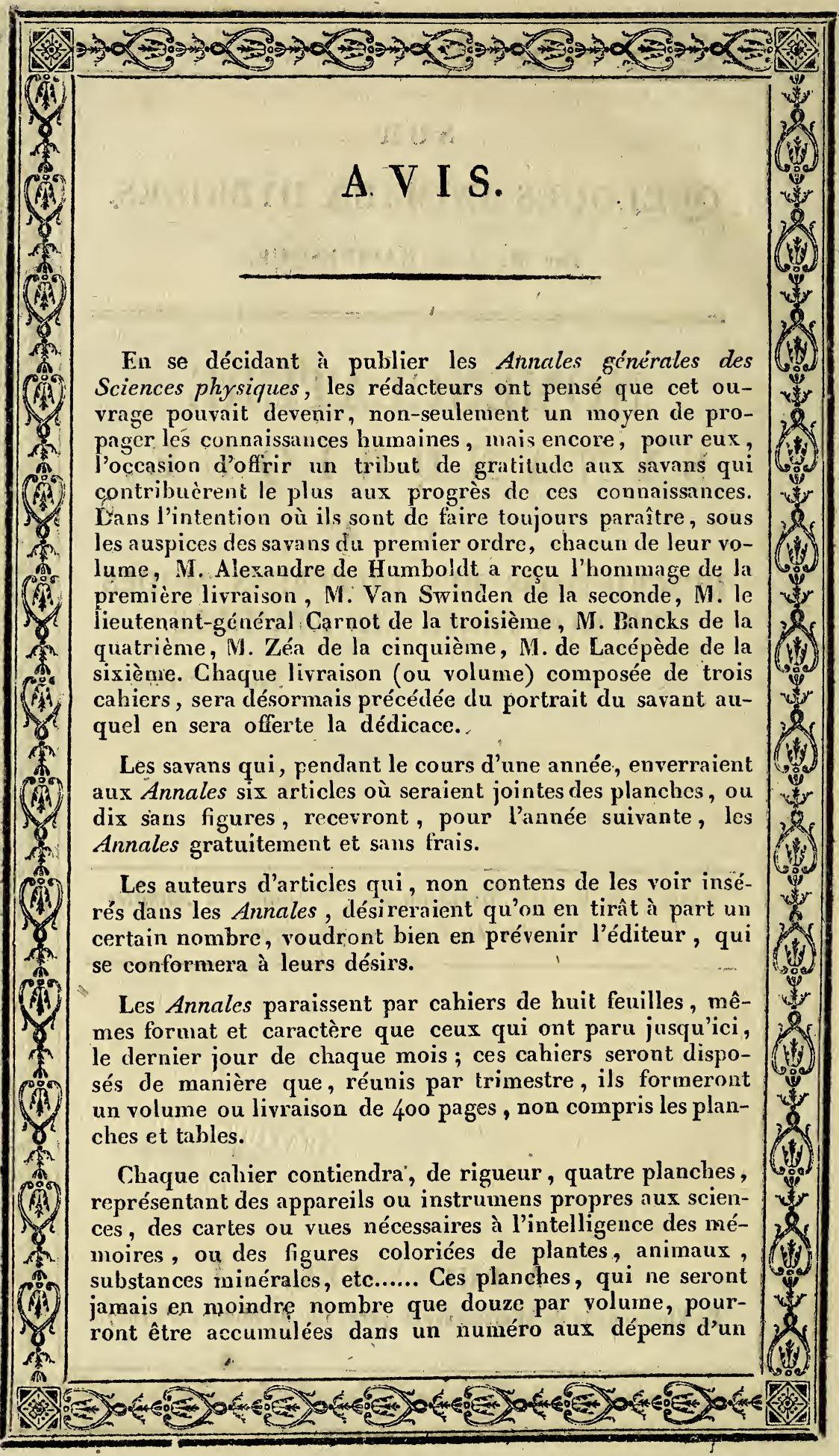




\title{
SUR QUELQUES ANIMAUX HYBRIDES.
}

\author{
Par M: C. S. RAFINESQUE,
}

Professeur de botanique et d'histoire naturelle dans

l'Université de Lexington en Kentuky.

L'hybridité animale est un des plus singuliers phénomènes zoologiques; elle nous prouve surtout que nous sommes bien Join de connaitre tous les faits qui ont rapport à l'histoire naturelle des amimatix. II est à-peuprès reçu maintenant comme axiôme, par les naturalistes, que les seuls quadrupèdes analogues et de même genrè peuvent produire des mulets. Je vais étahlir, par deux faits nouveaux, en contradiction avec celte règle, des ex-ceptions bien remarquables; $j$ 'en indiquerai en outre quel-ques autres qui pourront également conirarier un autre axiôme généralement admis, savoir : que les animaux quî produisent des mulets féconds sont toujours d'espèce identique.

Voici le premier fait qui est bien avéré. Une chatte fut laissée dans une cabane dans les bois du Kentuky, laquelle fut abandonnée pendant plusieurs mois. Cette cabane était partaitement isolée et éloignéc de plusieurs lieues de toute autre, et il n'y avait pas de chats dans le voisinage à la distance de 15 à is milles. Le propriétaire de la cabane trouva, à son retour, sa chatte encore dans la cabane et allaitant une portée de cinq petits monstres, semblables aux chats par le corps et le poil, mais ayant la tête, les pattes et la queue semblables à ceux du didelphe commun des ltats-Unis, nommé ici Opossum (le Didelphis Virginianus des naturalistes). Ces animaux vécurent et furent montrés comme curiosité dans tousles environs; mais ils sont morts jeunes et sans s'être propagés. On a conjecturé avec fondement que cette chatte, ainsi isolée, abaudonnée, et qui a vécu d'oiseaux, de souris 
et de taupes dans l'intervalle, aura agacé un didelphe mâle durant la période ordinaire de chaleur, à défaut de mâle d'espèce analogue, car il n'y a pas même de chats sauvages dans le Kentuky, (ceux qu'on nomme ainsi sont des lynx) et aura été fécondée par lui. Nais il est bien singulier qu'une union entre des animaux aussi différens, puisqu'ils appartiennent non-seulement à des genres, mais même à des familles et à des ordres dissemblables, ait été productive; la seule analogie entr'eux consiste peut-être dans une similitude de taille.

Le second fait quoique moins avéré, ne laisse cependant que quelques faibles doutes. Les chassears aborigènes de l'A mérique septentrionale croient que le raton ou raccoon (Procyon Loter, des naturalistes), peut s'unir avec le renard ronge. Il y a deux espèces au moins de ce nom à l'est du Mississipi (et plusieurs au-delà), le Canis virginianus, et mon Canis leucurus qui diffère du précédent par sa queue blanche, etc., je l'ai décrit dans Western revieuw, vòl. I, pag. 236 ; tous deux ont long-temps été confondus sous le nom de Canis vulpes. J'ai vu la dépouille complète d'un des produits de cette union; il ressemblait beaucoup plus au raton qu'au renard; il en avait la tête, les dents, les pattes, etc.; mais il avait l'encolure, la taille, et la couleur du renard rouge, à queue noire. La bande noire du visage, si remarquable dans le raton, avait disparu; elle était remplacěe par une faille teinte foncée : il en était de même des anneaux noirs de la queue. Il est donc probable que cet animal, qui était parfaitement adulte, avait été produit par l'union d'un raton avec un renard; animaux tous deux sanvages, et de genre, famille et ordre différens; cependant l'ou pourrait soupçonner que c'était peut-être une simple variété du raton, si le témoignage des aborigènes qui connaissent si bien les míeurs des animaux des forêts, n'élait contraire à cette opinion.

'A l'égard des mulets féconds, il est reconnu depuis long-temps que le bison d'Amérique (Taurus americanus des 


\section{( 89 )}

naturalistes ), ne peut produire avec le taureau et la vache domestiques, et Buffon s'est prévalu de ce fait pour conclure que ces especes sont idertiques, nonobstant que ces añimaux diffèrent bien plus entr'eux que l'âue et le cheval ou le buffle et le taureau. Le bison d'Amérique qui était commun dans toute l'Amérique septentrionale et particulièrement en Kentuky il y a 30 et 40 ans, a maintenant entièrement disparu de toute la région à l'orient du Mississipi, où il était trop harcelé; on le trouve encore en immenses troupeaux daus les vastes prairies ou stepes des plaines du haut Missouri ; cependant il se retire continuellement vers l'occident et le nouveau Mexique, à mesure qu'on le harcèle avec les armes à tea. Au reste cet utile animal, qui fournit ane chair délicate of une si belle toison, commence à exister en domesticité dans le Kentucky et dans plusieurs antres états occidentaux. Il est devenu presqu'aussi traitable que le taureau et la vache, il aime leur compagnie, et s'unit sans difficulté avec la vache, tandis que le taureau domestique a souvent de la répugnance pour la femelle du bison. Les métis qui en résultent, nommés en anglais Naalf-breed Buffalves, participent des denx animaux, obtenantla forme de la vache, mais conservent la couleur, et la tête du bison, ainsi qu'une demi-toison; ils perdent la bosse, mais ils ont encore le dos incliné. Ils s'unissent indifférencmententr'eux ou avec leurs pères et mères, produisent des nouvelles races et fournissent du bon lait comme la vache ( $\mathrm{x}$. Nonobstant ces faits, il me semble bien difficile de croire que les bisons d'Amérique soient identiques avec nos vaches et nos taureaux : ils en diffèrent par le squelette, la tête, les cornes, la barbe, la crinière, la toison, la basse, le dos incliné, la queue courte, etc. Ne serait-il pas passible que des espèces originairement différentes, donnassent dans certaines circonstances, des produits féconds?

(1) La gestation et partuition do la vache couverte par le bison, est plas pénible qu'à l'ordiraire. 


\section{$(90)$}

Nous en avons déjà quelques exemples dans le renard et lo elịien, l'âne et le zèbre, etc., et plusieurs parmi les oiseaux, auxquels je vais en ajouter un nouveau, qui s'ap: plique parfaitement au cas en question, à moins que l'on he veuille admettre une plus grande facilité d'union et un plus grand penchant à la fécondité, parmi les oiseaux que parmi les quadrupèdes.

L'oie du Canada ou oic à cravatte (Anser canadensis) a été complètement apprivoisée dans les Etats-Unis, où elle exxiste en parfaite domesticité. Elle s'est uniè presqu'aussitôt avec l'oie domestique, et a produit des indivịdus féconds, lesquels ont produit de poureaux métis féconds, par le croisement des races; ils participent plus ou moins de la naturre des espèces dont ils proviennent, à mesure qu'ils s'éloignent des types originels. Cependant l'oie à cravatte été regardée comme une espèce distincte par tous les naturalistes, et mếme par' Buffon, quoịu'il fût si porté à restreindre le nombre des espéces par esprit de système.

La mêtme union féconde a lieu entre le canard domestique et le canard musqué, quoique ce dernier diffère si essentiellement du premier, qu'il devrait peutêtre former un genre, ou sous-genre à part, à cause surtout de sa tête caronculée. Is diffèrent entr'eux cerțainement autant ou plus que les aras ne different des merroquets dont ils out été séparés. 
Nomenclature synandrique ou Descriptions des DIFFÉRENS MODES D'UNION PARMI LES ÉTAMINES.

Par C. S. RAFINESQUE, professeur de botanique, etc. à Lexington en Kentuky.

L'union des étamines offre des caractères importans, dont Linné s'est même souvent prévalu, pour former des classes et des ordres dans son système sexuel; mais les modes nombreux qu'elle assure n'ont pas tous été décrits par le̊s botanistes, ni considérés dans leur ensemble, ni đénommés convenablemént. Ôn n'emploie encore que les noms Linnéens, de Diadelphe, Monadelphe, Polyadelphe et Syngenèse, qui ne sont pas toujours appliqués à propos. Cependant rien n'est plus utile que de donner des noms convenables à toutes les modifications organiques, cela les isole, les fixe dans la mémoire, et évite l'emploi de longues périphrases. C'est dans ces vues que je me propose de suppléer à cet oubli, en indiquant toutes les différentes manières d'union staminales qui me sont connues, et en offrant un tableau synoptique.

La Synandrie ou union staminale en général, doit se diviser en trois branches : L'organe qui en résulte doit se nommer Synandre.

I. La Synopantie, ou union complète des filamens et des anthères.

II. La Synémie, ou union des filamens.

III. La Synanthérie, ou union des seules anthères.

Comme la Synopantie offre absolument les mêmes modifications synandriques que les deux autres branches, mais réunies dans la même fleur, il suffira d'analyser celles des filamens et des anthères considérées séparément. La désignation synopantique sera donc toujours double, par exemple Monadelphe-Synocoile, Synostyle-Syuostère, etc. 


\section{Synémie ou Synostémie.}

1. Monadelphie. Union des filamens en un seul corps : ce corps a reçu-de Nirbel le nom d'Andryphore, qui est impropre, car il signifie porte-étamine au lieu de porteanthère, et doit être réservé au support des étamines : je propose de le nommer Synostème, ce qui est plus doux à l'oreille, que le terme Symphyostemon de Moeuch. Tous deux signifient filamens unis.

A. Stŕrźadelphie. Union cọmplète en un corps solide. Cette union n'existe guèrẹs que dans les fleurs mâles; le corps qui en résulte pourra se nommer Synostème solide ou Stéréostème.

1. Pharosynie. Union en forme de tubercule ou sphère, etc., comme dans le genre Taxus, etc.

2. Synostylie ou Styladelphie. Union en förme de colonne cylindrique, comme dans les genres Stylidium, Hura, Xylophyllum, Acalypha; etc.

3. Némadelphie. Union en forme de fil. Dans quelques Euphorbiacées.

4. Gonadelphie. Union en corps anguleux.

5. Stéréabașie. Filamens unis seulement à leur base en un Stéréostème.

6. Stéréoxie. Le contraire ou filamens unis ensemble au sommet en un corps solide.

7. Synadelphie. A Stéréostème divisé régulièrement en des Stéréostèmes secondaires. Toutes ces différentes modifications se trouvent dans plusieurs fleurs monoïques et dioïques.

8. Cladelphie. A Stéréostème ramifié irrégulièrement plus ou moins de fois. Exemple licinus.

9. Stégynie. A Stéréostème formant un corps solide qui recouvre le pistil, comme dans les Asclépiadées.

yo. Gynandrie. A Stéréostème soudé en un seul corps quec le pistil, comme dans les Orchidées.

3. Périader phe. Union des filamens en forme circulaire 
autour du pistil. L'organe qui en résulte pourra se nomamer Synostème circulaire ou Péristème.

11. Siphadelphie. Union en forme de tube cylindrique. C'est le mode le plus commun, et que l'on nomme communément pour cela Monadelphe.

12. Diplosiphie. Union en double tube. Rare; quelques Méliacées.

x3. Urcéolinie. Union urcéolée, portant souvent les anthères intérieurement. Méliacées, etc.

14. Corollinie. Union formant un Péristème corolliforme, comme dans les genres Guara, Gomphrena, Pancratium, etc.

15. Annulinie. Péristème en forme d'anneau, comme dans le genre Anacardium.

16. Basadelphie. Union des filamens à leur base, comme dans les genres Linum, Geranium, Oxalis, Tamarix, Passiflora, Lysimachia, etc.

1 7. Mésadelphie. Union des filamens par leur milieu seulement; union rare.

18. Oxadelphie. Union par leur sommet seulement, comme dans beaucoup de fleurs radiées.

I9. Stéphadelphie. Union en forme de couronne.

20. Oxodontie. Union en forme de tube ou coupe, qurmonté par des dents.

21. Cyathonie. Union en forme de godet ou coupe à bords entiers.

2.2. Panadelphie. Cela exprimera une union intime ou complète quelconque. Peut-être pourrait-on en faire un ordre particulier.

23. Adelphémie. De mêmè, quand l'union n'existe qu'à demi, et

24. M'́radelphie. Quand elle n'existe qu'en partie.

25. Alternosie. Union où les parties désunies sont alternes avec des appendices.

26. Dianonie. Quand il y a des parties désunies et divisées.

87. Hetéradelphie, Quand ces parties sont inégales, 


\section{$(94)$}

28. Fénestrosie. Union partielle des filamens, laissant des intervalles désunis; tous ces modes, se retrouvent dans plusieurs genres, mais ce dernier est très-rare.

C. Pleuradelphie. Union circulaire interrompue ou latérale. L'organe qu'elle forme pourra se nommer $s_{y-}$ nostème latéral ou Pleurostème.

29. Schizadelphie. Union en forme de tube fondu latéralement. Quelques légumineuses, etc.

3o. Platadelphie. Union formant une surface plane latérale.

3r. Plagiadelphie. Union oblique.

32. Otadelphie. Union auriculée, ou en forme de cornet, auricule, etc.

33. Diarésynie. Union latérale divisée au moins jusqu'au milieu.

34. Synomérie. Union basillaire.

35. Cheiradelphie. Union palmée ou digitée. Ces modes d'union sont rares et singuliers.

D. Monasynie. Union des filamens en un seul corps, avec un ou plusieurs autres filamens désunis. Les filamens unis peuvent présenter tous les modes d'union, indiqués cidessus, tandis que considérés conjointement avec les fila: mens libres, ils peuvent former les résultats suivans.

36. Siphonie. Plusieurs filamens réunis et un libre for mant un tube. La majeure partie de la Diadelphie de Linné y appartient, n'étant nullement diadelphes dans le vrai sens de ce mot, surtout les légumineuses décandres.

37. Eltromonde. Plusieurs filamens réunis et un libre ne formant pas de tube. Cette réunion peut se sous-nommer, Dimonie, Trimonie, Tétranronie, Pentamonie, Polymonie, etc. selon que le nombre des filamens réunis sera de $2,3,4,5$ ou plusieurs. La Diadelphie décandrie de Linné serait dans ce cas une Ennémonie outre une Siphonie.

38. Ellrodie. Plusieurs filamens réunis et 2 libres. Sousdénominations Didie, Tridie, Tétradie, Pentadie, Polydie, ete. 


\section{$\left(9^{5}\right)$}

3g. Eltrie on Eleuthérotrie. Trois filamens libres, Ditrie, Tritrie, Polytrie, étc.

40. Eltropolie. Plusieurs ou plus de 3 filamens libres. Si l'on veut continuer ces dénominations on aura $E l$ trotétrie, Eltropentie, Eltrexie, Eltréptie, Eltroctie, Eltrennie, Eltrodecie, etc., avec leurs sous-dénominations respectives. Celles de l'Eltropolie sont la Dipolie, Tripolie, Tétrapolie, Pentapolie, Polypolie, etc.

II. Synopolie. Union des filamens en deux ou plasieurs corps. Ces corps devront aussi sé nommer Synostèmes.

E. Isadelphie. Deux ou plusieurs synostèmes égaux eţ à nombre égal d'étamines.

4r. Diadelphie. Deux unions, comme dans le genre Fumaria, etc. Considérée d'après les formes des unions ou synostèmes, elle admet pour sous-dénominations toutes les sortes d'unions monadelphiques et pleuradelphiques; tandis que considérée d'après le nombre des étamines elle est tétrandrique, exhandrique, octandrique, polyandriquề, etc.

42. Triadelphie. Trois unions, comme dans les genres Hypericum, Elodea, Triadenum, etc. Sous-dénominations numériques : Ennéandrique, Dodécandrique, Polyandrịque, etc.

43. Tétradelphie. Quatre unions égales.

44. Pentadelphie. Cinq unions égales, comme dans les genres Androsemum, Loasa, etc.

45. Polyadelphie. Plus de 5 unions égales, etc.

F. Symallie. Deux ou plusieurs unions inégales.

46. IMorphallie. Unions inégales en formes seulement: On pourra exprimer ces formes par les désignations déja employées pour les unions solitaires.

47. Rithmallie. Unions inégales en nombres seulement. Elles donneront lieu à plusieurs désignations secondaires, telles que Ditrosynie 2 et 3 étamines. Ditétrynie, 2 et 4. Dipentynie, 2 et 5. Dipalynie, 2 et plusịeurs. Tritétrynie, 3 et 4. Tripentynie, 3 et 5 . Tripolynie, 2 et plusieurs. 
Tétrapentynie, 4 et 5. Tétrapolynie, 4 et plusieurs. Pentapolynie, 5 et plusieurs. Anomalinie, nombres variables. Oligosynie, peu d'unions inégales. Polysynie, plusieurs unions inégales, Ditripolie, etc.

48. Diplallie. Unions inégales en formes et en nombres. Elles donneront lieu à beancoup de désignations secondaires, sur le plan proposé.

G. Chorizrnie. Deux unions ou plusieurs, avec un , deux ou plusieurs filamens séparés ou désunis.

49. Disynisie. Deux unions égales. Dénominations secondaires Monochorizie, Dichorizie, Trichorizie, Polychorizie, etc. suivant qu'il y a $x, 2,3$ ou plusieurs filamens désunis.

5o. Trisynisie. Trois unions égales. Dénominations secondaires comme ci-dessus.

5x. Polysynisie. Plasieurs unions égales ou plus de trois, etc. Tétrasynisie, Pentasynisie, etc.

52. Disynétrie. Deux nnions inégales en nombre ou forme, ou l'un et l'autre, etc.

53. Trisynétrie. Trois unions inégales, dito., etc.

54. Polysynétrie. Plus de trois unions in égales, dito.

II. Synanthérie.

I. Synomonie. Union des anthères en un seul corps qui doit se nommer le Synanthère.

A. Synostérie. Union formant un Synanthère solide ou non tubuleux ni perforé.

I. Endostérie. Synanthère complètement solide.

2. Synocoilie. Synanthère creux ou caverneux.

3. Pettosynie. Synanthère en forme de bouclier ou pelté.

4. Calyptrosie. Synanthère en forme de calyptre ou coëffe.

5. Synactie. Synanthère en forme d'étoile, Siyrosynie en forme de croix.

6. Géminie. Deux anthères géminóes ou unies à leur base.

7. Triclosynie. Anthères réunies par des poils, comme dans quelques Labiées, etc.

8. Oxynie. Anthères légérement réunies par leur sommet. 


\section{(97)}

9. Pleurosynie. Anthères réunies latéralement.

10. Synocormie. Anthères réunies en massue ou colonne.

B. Périsynie. Union circulaire, à centre et à sommet perforé.

11. Siphosynie. Synanthère tubuleax, comme dans les fleur's flosculeuses, etc.

12. Trochosynie. Synanthère en forme de roue.

13. Cérosynie. Synánthère en forme de corne.

14. Odosynie. Synanthère tubuleux à sommet denté ou appendiculé.

15. Gonosynie. Synanthère anguleux, tubuleux.

16. Pleuropérie. Anthères légérement unies ou conniventes latéralement, comme dans les G.Viola, Solanum, etc.

11. Polysiz. Anthères réunies en deux ou plusieurs corps ou synanthères. Leurs formes auront les mêmes dénominations que les précédentes.

C. Isxine. Synanthères égaux en formes et nombres d'étamines.

7. Disynie. Deux synanthères : chacun desquels peut être diandre, triandre, etc.

18. Trisynie. Trois synanthères, etc.

19. Tétrasynie. Quatre synanthères, etc.

20. Pentasynie. Cinq synanthères, etc.

21. Polysynie. Plusieurs synanthères, etc.

D. Alcosyne. Synanthères inégaux en formes ou nombres, ou l'un et l'autre.

22. Anisosynie. Inégalité de nombre d'anthères seulement. Désiguations secondaires, les mêmes que pour la synostémie rithmallie 47 .

23. Anisorphie. Inégalité de formes synanthériques.

24. Diplanisie. Inégalités de nombres et de formes.

Toutes ces modifications d'unions synanthériques, existent dans quelques genres de plantes, mais on! a négligé de les indiquer; il n'y en a qu'un petit nombre que j'ai ajouté par supposition, et je suis certain qu'on les retrouvera un jour, parmi les plantes tropicales, dès qu'on les examinera sous ce point de vue. 






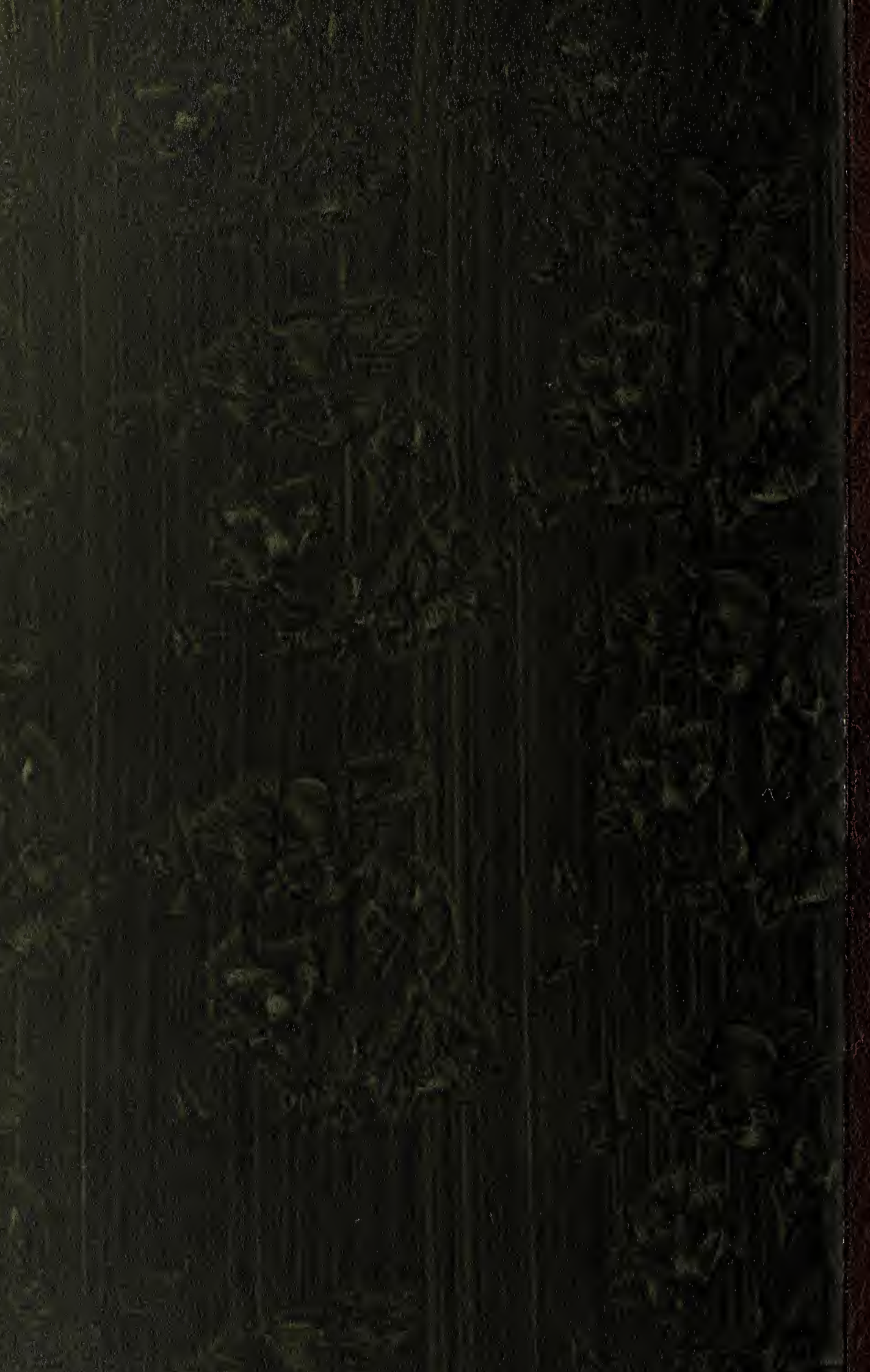

